Pacific Journal of Mathematics

MINIMAL MEASURED LAMINATION IN GEOMETRIC 


\section{MINIMAL MEASURED LAMINATIONS IN GEOMETRIC 3-MANIFOLDS}

\section{KEN'ICHI OHSHIKA}

In this paper we deal with codimension-1 measured laminations whose leaves are minimal surfaces in geometric 3-manifolds with either $\widetilde{S L_{2}} \mathbf{R}$ or $\mathbf{H}^{2} \times \mathbf{E}$ structures. We call such measured laminations minimal measured laminations. Our main theorem states that in a geometric 3-manifold with an $\widetilde{S L}_{2} \mathbf{R}$-structure every class in $\mathbf{R}^{\mathscr{S}}$ containing incompressible measured laminations is represented uniquely by a minimal measured lamination. This implies that every incompressible lamination in such a 3-manifold is equivalent to a unique minimal measured lamination, which is vertical with respect to geometric fibering structure.

Introduction. Our subjects in this paper are minimal measured laminations in geometric 3-manifolds i.e. measured laminations whose leaves are minimal surfaces in geometric 3-manifolds. Our main result is concerning minimal laminations in 3-manifolds with $\widetilde{S L_{2}} \mathbf{R}$ or $H^{2} \times E$-structures. It is shown in Hass [4] that in a geometric 3-manifold with an $\widetilde{S L_{2}} \mathbf{R}$-structure, every incompressible surface is isotopic to a minimal incompressible surface and that every incompressible minimal surface is a vertical torus. Our main theorem is a generalization of this theorem by Hass to incompressible measured laminations. Our main theorem for geometric 3-manifolds with $\widetilde{S L_{2}} \mathbf{R}$-structures is as follows.

THEOREM 3.1. Let $M$ be a 3-manifold with a fixed $\widetilde{S L_{2}} \mathbf{R}$-structure $g$. Let $m$ be a class of $\mathbf{R}^{\mathscr{S}}$ represented by an incompressible measured lamination in $M$. Then there exists a unique incompressible minimal measured lamination $L$ in $(M, g)$ which represents $m$. Moreover $L$ is vertical with respect to geometric fibration of $(M, g)$.

In the case of geometric 3-manifolds with $H^{2} \times E$-structures, we need an additional assumption as follows.

THEOREM 3.2. Let $M$ be a Seifert fibered manifold with a fixed $H^{2} \times$ E-structure $g$. Let $m$ be a class of $\mathbf{R}^{\mathscr{S}}$ represented by an incompressible measured lamination such that $m(h)=0$ where $h$ 
denotes the class of regular fibers. Then there exists a unique incompressible minimal measured lamination $L$ in $(M, g)$ which represents $m$. Moreover $L$ is vertical with respect to geometric fibration of $(M, g)$.

We obtain the following corollary from the theorem above.

COROllary 3.3. Let $(M, g)$ be as in Theorem 3.1. Then every incompressible measured lamination is equivalent to a unique minimal measured lamination. Moreover every minimal measured lamination is a vertical saturated set over some geodesic measured lamination on the base 2-orbifold $O$.

1. Preliminaries. Throughout this paper we deal with closed smooth 3-manifolds. By a word lamination we always mean codimension-1 lamination. A lamination $L$ is a closed subset of a manifold $M$ satisfying the following condition. There exists a local chart $\left\{U_{i}\right\}$ where $U_{i}=V_{i} \times I$ for some open subset $V_{i}$ of $\mathbf{R}^{2}$, such that $L \cap U_{i}=V_{i} \times X$ for some closed subset $X$ of $I$, and such that for $U_{i}$ and $U_{j}$, the coordinate exchange map $\phi_{i, j}$ can be factored to a coordinate exchange map between $V_{i}$ and $V_{j}$ and that of $I$. For details of the definition of laminations in general see Morgan-Shalen [5]. A measured lamination is a lamination equipped with an invariant transverse measure.

A branched surface is a $C^{1}$-surface with one-dimensional branching singularities which may intersect mutually transversely at triple points. A fibered neighbourhood $N(\mathscr{B})$ of a branched surface $\mathscr{B}$ is a neighbourhood of $\mathscr{B}$ fibered by intervals. A branched surface $\mathscr{B}$ is said to be incompressible when the following three conditions are satisfied.

(i) The horizontal boundary of the fibered neighbourhood $\partial_{h} N(\mathscr{B})$ is incompressible in $M-\stackrel{\circ}{N}(\mathscr{B})$.

(ii) There are no disks of contact.

(iii) There are no monogons.

See Floyd-Oertel [3] for details.

A measured lamination $L$ is said to be carried by a branched surface $\mathscr{B}$ when $L$ can be isotoped so that $L$ is contained in $N(\mathscr{B})$ transversely to all fibers of $N(\mathscr{B})$. A measured lamination is said to be incompressible when it is carried by an incompressible branched surface.

Let $(M, g)$ be a manifold with a Riemannian metric $g$. A lamination $L$ is said to be minimal (with respect to $g$ ) when all its leaves 
are minimal surface (with respect to $g$ ). We will deal with minimal lamination in 3-manifolds with $\widetilde{S L_{2}} \mathbf{R}$ or $H^{2} \times E$-structures.

Throughout this paper the symbol $\mathscr{S}$ denotes the set of free homotopy classes of loops in $M$. The symbol $\mathbf{R}^{\mathscr{S}}$ denotes the set of all functions from $\mathscr{S}$ to the set of real numbers $\mathbf{R}$. A measured lamination $(L, \mu)$ corresponds to an element $[L, \mu]$ of $\mathbf{R}^{\mathscr{S}}$ by the equation $[L, \mu](s)=\inf _{\sigma \in s} \mu(\sigma)$ for $s \in \mathscr{S}$ where the left hand of the equation denotes the $s$-coordinate of $[L, \mu]$.

Two measured laminations $L_{1}$ and $L_{2}$ are said to be equivalent if they are carried by a branched surface with the same weights. This implies that the two measured laminations are isotopic except for their compact leaves.

The following lemma is often used in the proof of our main theorem.

LEMMA 1.1. Let $(L, \mu)$ be a measured lamination in a closed 3manifold $M$, none of whose components are closed surfaces. Then no leaves of $L$ are isolated in $L$. Moreover for any $\varepsilon>0$ there exists $\delta$ such that for any interval $I \subset M$ whose length is less than $\delta, \int_{I} \mu<\varepsilon$.

Proof. Let $l$ be an isolated leaf of $L$. As $l$ is contained in the support of $\mu, l$ has an atomic measure. As $M$ is closed this is possible only when $l$ is a closed surface.

As $(L, \mu)$ does not have leaves with atomic measures the latter part of the lemma easily follows from compactness of $M$.

2. Incompressible branched surfaces in geometric 3-manifolds. In this section we characterize incompressible branched surfaces in Seifert fibered manifolds with $\widetilde{S L_{2}} \mathbf{R}$ or $H^{2} \times E$-structures.

Proposition 2.1. Let $L$ be an incompressible measured lamination in a Seifert fibered manifold $M$ with $\widetilde{S L_{2}} \mathbf{R}$-structure. Then $L$ is carried by a vertical branched surface over a train track on the base orbifold $O$.

Proof of Proposition 2.1. By assumption $L$ is carried by an incompressible branched surface $\mathscr{B}$. By choosing an integral weight system $w$ on $\mathscr{B}$, the weighted branched surface $(\mathscr{B}, w)$ carries a union of incompressible surfaces $S_{1}, \ldots, S_{n}$. Such surfaces must be isotopic to vertical incompressible tori or vertical Klein bottles over simple closed curves on $O$ or vertical Klein bottles containing two singular 
fibers. Moving the branched surface by isotopy we assume that the surfaces $S_{1}, \ldots, S_{n}$ are vertical. If we choose $w$ vanishing on no sectors, the branched surface $\mathscr{B}$ can be constructed from $\bigcup_{i=1}^{n} S_{i}$ by identifying some subsurfaces of them. For $i \neq j$, let $P_{i, j}$ be the subsurface of $S_{i}$ which is identified with $P_{j, i}$ in $S_{j}$ to make the branched surface $\mathscr{B}$. When $i=j$, let $P_{i, i}^{1}, P_{i, i}^{2}$ be subsurfaces which are identified. (They may have common components.) We will show that we can retake $\mathscr{B}$ and $\left\{S_{i}\right\}$ so that $P_{i, j}$ is a vertical annulus or Möbius band for each $i, j$ preserving the condition that $\mathscr{B}$ carries the given measured lamination $L$ and that $\mathscr{B}$ is incompressible. Let $N(\mathscr{B})$ be a fibered neighbourhood of $\mathscr{B}$. We can assume the surfaces $\left\{S_{i}\right\}$ are embedded in $N(\mathscr{B})$ disjointly transversely to the fibers. Let $H_{\{i, j\}}$ be a cobordism in $N(\mathscr{B})$ between $P_{i, j}$ and $P_{j, i}$ (or $P_{i, i}^{1}$ and $P_{i, i}^{2}$ ) which is homeomorphic to an $I$-bundle over a surface or the quotient of a product bundle by an involution on the base surface $\times\{1\}$ in the case when $P_{i, j}$ is a double covering of $P_{j, i}$, and let $\Pi_{\{i, j\}}$ be the surface in $H_{\{i, j\}}$ corresponding to a lift of base surface of the $I$-bundle. Then $N(\mathscr{B})$ is decomposed as $\bigcup_{i} N\left(S_{i}\right) \cup \bigcup_{i \leq j} N\left(\Pi_{\{i, j\}}\right)$ so that they intersect each other only at their boundaries.

Note that if a component $P$ of $P_{i, i}^{1}$ is identified with itself, $P$ must doubly cover a surface $P^{\prime}$. As $P$ is equal to the boundary of an $I$-bundle over $P^{\prime}, P^{\prime}$ must be non-orientable. If an orientation reversing curve in $P^{\prime}$ were inessential in $M$, there would be an onesided projective plane embedded in $M$, which is impossible.

Now assume that there is some $P_{i, j} \quad(i \neq j)$ such that the image of $\pi_{1}\left(P_{i, j}\right)$ in $\pi_{1}\left(S_{i}\right)$ is trivial. Then $P_{i, j}$ is planar, and except one component $\alpha_{0}^{i}$ of $\partial P_{i, j}$, components $\alpha_{1}^{i}, \ldots, \alpha_{n}^{i}$ of $\partial P_{i, j}$ bound disks $D_{1}^{i}, \ldots, D_{n}^{i}$ in $S_{i}$. The same holds for $P_{j, i}$. We denote the component of $\partial P_{j, i}$ corresponding to $\alpha_{k}^{i}$ by $\alpha_{k}^{j}$ and the disk bounded by $\alpha_{k}^{j}$ in $S_{j}$ by $D_{k}^{j}$. The two simple closed curves $\alpha_{k}^{i}$ and $\alpha_{k}^{j}$ bound an annulus $A_{k}$ mutually disjointly. As $M$ is irreducible the sphere $D_{k}^{i} \cup A_{k} \cup D_{k}^{j}$ bounds a 3-cell $B_{k}$ in $M$. Then as $H_{\{i, j\}} \cup \bigcup_{k=1}^{n} B_{k}$ is also a 3-cell we can isotope $L$ in $M$ through $H_{\{i, j\}} \cup B_{k}$ so that $L \cap \Pi_{\{i, j\}}=\varnothing$ and $L$ is still contained in $N(\mathscr{B})$ transversely to fibers (Figure 2-A). Then we deform $B$ by removing $H_{\{i, j\}}$ from $N(\mathscr{B})$. It is obvious that the branched surface constructed above is still incompressible and carries $L$.

In the following, either $P_{i, j}$ is homeomorphic to $P_{j, i}$ or one doubly covers the other. As the latter case can be treated by the same way as the former case, for simplicity we only deal with the former case. 

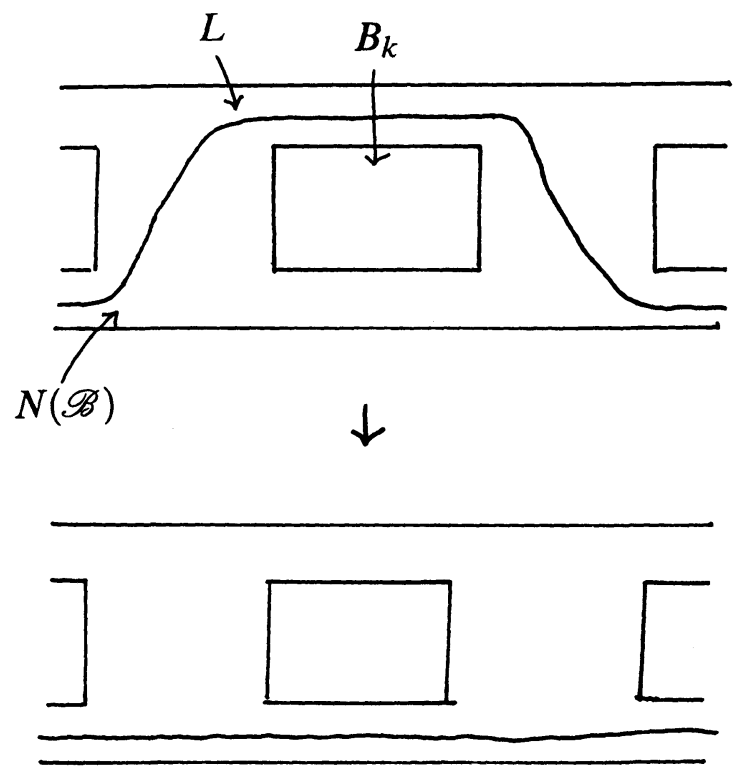

FIGURE 2-A

Next, suppose that there is some $P_{i, j}$ such that some of the boundary components are inessential and the image of $\pi_{1}\left(P_{i, j}\right)$ in $\pi_{1}\left(S_{i}\right)$ is non-trivial. As before inessential components $\alpha_{1}^{i}, \ldots, \alpha_{n}^{i}$ and $\alpha_{1}^{j}, \ldots, \alpha_{n}^{j}$ bound disks $D_{1}^{i}, \ldots, D_{n}^{i}$ on $S_{i}$ and $D_{1}^{j}, \ldots, D_{n}^{j}$ on $S_{j}$ respectively. We can number the $\alpha_{k}^{j}$ 's so that $\alpha_{k}^{i} \cup \alpha_{k}^{j}$ bounds an annulus $A_{k}$ and the sphere $D_{k}^{i} \cup A_{k} \cup D_{k}^{j}$ bounds a 3-cell $B_{k}$. Then we construct a new $N(\mathscr{B})$ by letting it be equal to $N(\mathscr{B}) \cup \bigcup_{k} B_{k}$. Then it is obvious that the new branched surface $\mathscr{B}$ still carries $L$ and is incompressible. Moreover by this method we can make every component of $\partial P_{i, j}$ essential for the new branched surface $\mathscr{B}$.

Next, suppose that every component of $P_{i, j}$ is essential for each $i, j$. Then each component of $P_{i, j}$ is an annulus or Möbius band in the case when $S_{i}$ is a Klein bottle. Assume moreover that a component $\alpha_{i, j}$ of $\partial P_{i, j}$ is not homotopic to a regular fiber of $M$. Then as both $\pi_{1}\left(S_{i}\right)$ and $\pi_{1}\left(S_{j}\right)$ contain the homotopy classes represented by $\alpha_{i, j}$ and a regular fiber, $S_{i}$ and $S_{j}$ are isotopic (or $S_{j}$ doubly covers $S_{i}$ ). Let $T_{i, j}$ be the cobordism in $M$ between $S_{i}$ and $S_{j}$. Then we let a new $N(\mathscr{B})$ be equal to $N(\mathscr{B})$. The new $\mathscr{B}$ is obviously incompressible and carries $L$. Doing this for all the $P_{i, j}$ 's, we can assume that every component of $P_{i, j}$ is a vertical annulus for each $i, j$ if $i \neq j$. 
In the case when $\partial P_{i, j}$ is empty, either $S_{i}$ or $S_{j}$ is unnecessary to make $\mathscr{B}$ so we can remove one of them.

Finally we consider the case when $i=j$. We again assume that $S_{i}$ is a torus for simplicity (the case when $S_{i}$ is a Klein bottle is quite similar). Let $A$ be a component of $P_{i, i}^{1}$ which is identified with $B \subset P_{i, i}^{2}$ different from $A$. By the same argument as the case when $i \neq j$, we can assume that $A$ is an annulus. Let $C_{1}$ and $C_{2}$ be the closures of two components of $S_{i}-(A \cup B)$. Then connecting two boundary components of $C_{l}(l=1,2)$ in $H_{\{i, j\}}$ we can make two tori $T_{1}$ and $T_{2}$. If one of them is compressible, $\mathscr{B}$ either contains monogon or carries a compressible surface which is impossible. Then the homology class of $S_{i}$ must be the sum of two classes represented by the incompressible tori, which is possible only when $A$ is homotopic to a vertical annulus.

Next assume that a component $A$ of $P_{i, i}^{1}$ is identified with itself. This can be possible only when $\pi_{1}(A)$ contains the homotopy class of a regular fiber as we have seen before. If neither $A$ is an annulus nor $\partial A=\varnothing, \partial A$ contains inessential components and we can deform $\mathscr{B}$ as before and make $A$ an annulus or the whole of $S_{i}$. The case when $A=S_{i}$ happens only when $S_{i}$ is a double covering of a vertical Klein bottle $K_{i}$. Then we replace $S_{i}$ by $K_{i}$. Anyway $P_{i, j}$ is made vertical preserving the condition that $\mathscr{B}$ is incompressible and carries $L$. Then $\mathscr{B}$ is also isotopic to a vertical branched surface over a train track on $O$ possibly having complementary regions which are annuli, Möbius bands, or digons. It is easy to remove these complementary regions by identifying the branches bounding them with their axes. (In the case of digons we call the segments inside of them connecting their angles "axes".)

By the same argument we can prove the following.

Proposition 2.2. Let $L$ be an incompressible measured lamination in a Seifert fibered manifold $M$ with $H^{2} \times E$-structure such that for a regular fiber $h, i(L, h)=0$. Then $L$ is carried by a vertical branched surface over a train track on the base orbifold $O$.

The following corollary easily follows the propositions above.

CoROllaRy 2.3. Let $L_{1}, L_{2}$ be incompressible measured laminations in $M$ as in Proposition 2.1 or 2.2. Suppose that $\left[L_{1}\right]=\left[L_{2}\right]$ in $\mathbf{R}^{\mathscr{S}}$. Then $L_{1}$ and $L_{2}$ are equivalent. 


\section{Minimal measured laminations in 3-manifolds with $\widetilde{S L_{2}} \mathbf{R}$-struc-} tures. In this section we prove our main theorem.

THEOREM 3.1. Let $M$ be a 3-manifold with a fixed $\widetilde{S L_{2}} \mathbf{R}$-structure $g$. Let $m$ be a class of $\mathbf{R}^{S}$ represented by an incompressible measured lamination in $M$. Then there exists a unique incompressible minimal measured lamination $L$ in $(M, g)$ which represents $m$. Moreover $L$ is vertical with respect to geometric fibration of $(M, g)$.

Proof of Theorem 3.1. In the following, we mean "vertical with respect to the geometric fibration" by the word "vertical" for surfaces or laminations. First we prove the existence of a minimal lamination $L$ representing $m$. As $m$ is represented by some incompressible measured lamination $\Lambda$, there exists a weighted incompressible branched surface $(\mathscr{B}, \omega)$ carrying $\Lambda$. As is shown in Proposition $2.1, \mathscr{B}$ can be assumed to be a vertical branched surface. Hence $L$ is equivalent to a measured lamination carried by $(\mathscr{B}, \omega)$ which is a vertical measured lamination $L$ over a measured lamination on $O$. Obviously the measured lamination $L$ is minimal. Hence $m$ is represented by the minimal measured lamination $L$.

Next we will prove the uniqueness of $L$. Let $L$ be the vertical minimal measured lamination constructed above, and let $L^{*}$ be another incompressible minimal measured lamination representing $m$. We will prove that $L$ and $L^{*}$ are equal.

Since compact incompressible minimal surfaces in $M$ are vertical, as is shown in Hass [4], isolated leaves of $L^{*}$ are vertical. Let $L^{\prime}$ be the complement in $L^{*}$ of the set of all isolated leaves of $L^{*}$. We denote the transverse measures of $L, L^{*}, L^{\prime}$ by $\mu, \mu^{*}, \mu^{\prime}$ respectively. We will prove that $L^{\prime}$ is also vertical in the following. For that, we will prove that $L \cap L^{\prime}=\varnothing$. Let $\widetilde{L}$ and $\widetilde{L}^{\prime}$ be the lifts of $L$ and $L^{\prime}$ respectively to the universal cover $\widetilde{M} \cong \widetilde{S L_{2}} \mathbf{R}$. Let $q: \widetilde{M} \rightarrow M$ be the covering projection. Let $h$ be the element of $\pi_{1}(M)$ represented by a regular fiber of $M$.

Our first step is to prove that vertical tori approximating $L$ intersects $L^{\prime}$ only by simple closed curves homotopic to the regular fiber of $M$. Let $S$ be a vertical torus and let $\widetilde{S}$ be $q^{-1}(S)$. Suppose $\widetilde{S} \cap \widetilde{L}^{\prime} \neq \varnothing$. Let $\lambda$ be a component of $\widetilde{S}$ intersecting $\widetilde{L}^{\prime}$. We will analyze the type of the intersection $\lambda \cap \widetilde{L}^{\prime}$. We often use the following three facts. The first is that $\lambda \cap \widetilde{L}^{\prime}$ is invariant by a covering translation corresponding to $\pi_{1}(S)$. The second is the maximal principle of minimal surfaces that $\lambda$ can be tangent to $\widetilde{L}^{\prime}$ only in the form of the 
multiple saddle, the form $z=0$ and $z=\mathfrak{R}(x+i y)^{n}$. The third is that each component of $\lambda \cap \widetilde{L}^{\prime}$ is a properly embedded graph because $\widetilde{L}^{\prime}$ is carried by a branched surface.

First we see that there are no closed curves in $\lambda \cap \widetilde{L}^{\prime}$. If there were, we could find an innermost disk $D$ in $\lambda$ bounded by a simple closed curve $c \subset \lambda \cap \widetilde{L}^{\prime}$. Then the simple closed curve $c$ would bound a disk $D^{\prime}$ also in a component $\lambda^{\prime}$ of $\widetilde{L}^{\prime}$, and the 2-sphere $D \cup D^{\prime}$ bounds a 3cell in $\widetilde{S L_{2}} \mathbf{R}$. Hence $d\left(\lambda, D^{\prime}\right)$ is bounded. Let $z$ be a point of int $D^{\prime}$ where the distance function from $D^{\prime}$ to $\lambda$ attains the maximum. Then by translating $\lambda^{\prime}$ by an isometry $\xi$ along the perpendicular from $z$ to $\lambda$ we could make $\xi \lambda^{\prime}$ tangent to $\lambda$ from one side, which contradicts the maximal principle of minimal surfaces.

The plane $\lambda$ has a 2-dimensional Euclidean structure induced from the geometric structure $g$ in $M$. We choose a coordinate system so that the $y$-axis is a lift of a geometric fiber (i.e. $y$-axis is vertical).

We classify embeddings of $\mathbf{R}$ into $\lambda \cap \widetilde{L}^{\prime}$ into the following four types. Let $f: \mathbf{R} \rightarrow \lambda \cap \widetilde{L}^{\prime}$ be an embedding. We assume that the parameter is equal to the length parameter in $\lambda$.

1. The $x$-coordinate of $|f(t)|$ is bounded as $t \rightarrow \pm \infty$.

2. The $x$-coordinate of $|f(t)|$ is bounded as either $t \rightarrow-\infty$ or $t \rightarrow \infty$ and goes to infinity as $t$ goes to infinity with the other sign.

3 . The $x$-coordinate of $f(t)$ goes to infinity with the same sign when $t \rightarrow-\infty$ and when $t \rightarrow \infty$.

4. The $x$-coordinates of $f(t)$ go to infinity with different signs as $t \rightarrow-\infty$ and $t \rightarrow \infty$.

We will show that there can only exist an embedding of type (1) above. Moreover if an embedding of type (1) does exist, it must be $h$-invariant. Before proving these, we note the following fact. As $M$ is compact, there exists a constant $\eta$ such that for any point $x$ in $M$ there exists a flow-box for $L^{\left({ }^{\prime}\right)}$ centered at $x$ whose height with respect to $g$ is greater than $\eta$ everywhere. Let $\nu$ be a leaf of $\widetilde{L}^{\left({ }^{\prime}\right)}$. Then by lifting the flow-boxes above and taking a subset we can construct an $\eta$-neighbourhood $N_{\nu}$ of $\nu$ which has an $I$-bundle structure in the vertical direction of flow boxes, whose fibers are geodesic arcs orthogonal to $\nu$ with lengths equal to $\eta$ with respect to $g$ such that each leaf in the neighbourhood is transverse to the fibers. We call this neighbourhood the $\eta$-neighbourhood $N_{\nu}$ from now on.

(1) (The case when there exists an embedding $f$ of the type (1).) We assume that $f(t) \rightarrow \infty$ as $t \rightarrow \pm \infty$. First we will show that $f(\mathbf{R})$ must be invariant by the covering translation $h$. 
Assume that there exists an embedding $f: \mathbf{R} \rightarrow \lambda \cap \widetilde{L}^{\prime}$ of type (1) which is not invariant by the covering translation $h$. Let $f_{i}: \mathbf{R} \rightarrow \lambda \cap$ $\widetilde{L}^{\prime}$ be the composition of $h^{i}$ and $f$. Let $N_{f}$ be the $\eta$-neighbourhood of a component of $\lambda \cap \widetilde{L}^{\prime}$ containing $f(\mathbf{R})$. The images $f_{i}(\mathbf{R})$ 's may be contained in the same component of $\lambda \cap \widetilde{L}$ as $f(\mathbf{R})$. But by the assumption for sufficiently small or large $T$, the $f_{i}(\{t \mid t \leq T\})$ 's $(i=0, \pm 1, \pm 2, \ldots)$ or the $f_{i}(\{t \mid t \geq T\})$ 's are disjoint. We can assume the former holds since the argument is completely the same in the latter case as in the former case. The images $f(\mathbf{R})$ and $f_{i}(\mathbf{R})$ may intersect transversely. But by changing the track at these double points, we can assume that $f(\mathbf{R})$ does not intersect transversely the $f_{i}(\mathbf{R})$ 's $(i= \pm 1, \pm 2, \ldots)$.

Then if $y$-coordinates of $f(t)$ go to infinity with different signs as $t \rightarrow-\infty$ and $t \rightarrow \infty$, the situation of $\bigcup F_{i}(\mathbf{R})$ is as depicted in Figure 3.A. Then let $D_{i}$ be the space between $f_{i}(\mathbf{R})$ and $f_{i+1}(\mathbf{R})$. If the $y$ coordinates of $f(t)$ go to infinity with the same sign, let $E_{i}$ be the one of the open regions in $\lambda$ bounded by $f_{i}(\mathbf{R})$ whose $x$-coordinate is bounded. Then there are two possibilities. The one is the case when $\cdots \supset E_{-1} \supset E_{0} \supset E_{1} \supset \cdots$ (Figure 3.B-(a)). In this case let $D_{i}$ be $E_{i}-E_{i+1}$. The other is the case when $E_{i}$ 's are mutually disjoint (Figure 3.B-(b)). In this case take a line $\{x \mid x=K\}$ for sufficiently large $K$ so that it intersects all of the $f_{i}(\mathbf{R})$ 's, and let $D_{i}$ be a component of $\{x \mid x \geq K\}-\bigcup_{i} f_{i}(\mathbf{R})$ between $f_{i}(\mathbf{R})$ and $f_{i+1}(\mathbf{R})$ containing an end.

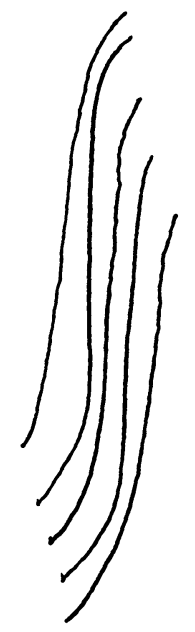

Figure 3-A 


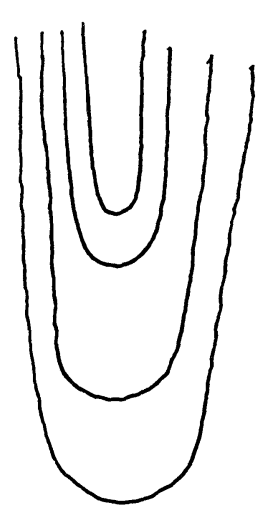

(a)

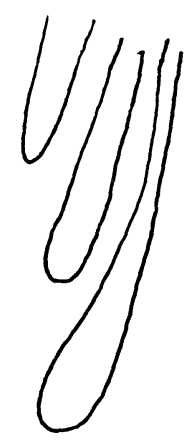

(b)

FIGURE 3-B

We will use the following claims later.

Claim 1. For any positive real number $\varepsilon$, there does not exist a sequence of embedded arcs $\left\{\alpha\left(s_{i}\right) \quad\left(0 \leq s_{i} \leq \varepsilon_{i}\right)\right\}$ in $\lambda \cap \widetilde{L}^{\prime}$ (where $s_{i}$ is the length parameter in $\lambda$ ) with a bounded sequence $\left\{\varepsilon_{i}\right\}$ such that $\varepsilon_{i} \geq \varepsilon$ and $d\left(\alpha(0), \alpha_{i}\left(\varepsilon_{l}\right)\right) \leq \delta_{i}$ where $\delta_{i} \rightarrow 0$ as $i \rightarrow \infty$.

Proof. Suppose that such a sequence exists. Then there exists a sequence $\left\{\beta_{i}\right\}$ of translates of the $\alpha_{i}$ 's by elements of $\pi_{1}(S)$ which stays in a compact subset of $\lambda$ because $\lambda / \pi_{1}(S)$ is compact. Then $\beta_{i}$ has a subsequence converging to a continuous map $\beta:[0, \delta] \rightarrow \lambda \cap \widetilde{L}^{\prime}$ with $\delta \geq \varepsilon$ and $\beta(0)=\beta(\delta)$. The image of $\beta$ cannot contain a closed curve as was shown before. Hence the image of $\beta$ must be a tree. As $\beta(0)=\beta(\delta)$, in a small neighbourhood of $\beta, \beta_{l}$ must turn to nearly the opposite direction at least once for large $i$. Let $\nu^{\prime}$ be a leaf of $\widetilde{L}^{\prime}$ containing the image of $\beta$. Let $N_{\nu^{\prime}}$ be the $\eta$-neighbourhoods of $\nu^{\prime}$. Then as the image of $\beta_{i}$ is contained in $\lambda \cap N_{\nu^{\prime}}$, such a situation is possible only when $\lambda$ is tangent to $\widetilde{L}^{\prime}$ from one side at the image of $\beta$, which contradicts the maximal principle of minimal surfaces.

We define a function $P$ from $f_{ \pm 1}(\mathbf{R})$ to the set of straight arcs in $\lambda$ as follows. For $x \in f_{ \pm 1}(\mathbf{R})$, the arc $P(x)$ is a perpendicular from $x$ to $f(\mathbf{R})$. We choose a perpendicular $P(x)$ so that the function $P$ satisfies the following condition. For $x \neq y$, two segments $P(x)$ and $P(y)$ do not intersect transversely. The length of $P(x)$ is smaller than the horizontal distance from $x$ to $f(\mathbf{R})$ in the same direction as $P(x)$. Let $t_{0}$ be a real number where $P \circ f_{ \pm 1}$ is not continuous. Then 
we require that $P \circ f_{ \pm 1}$ is left continuous at $t_{0}$ and $\lim _{t \rightarrow t_{0}+0} P \circ f_{ \pm 1}(t)$ also exists. Let $P^{\prime} \circ f_{ \pm 1}\left(t_{0}\right)$ denote the right limit. Then we require that $P^{\prime}\left(x_{0}\right)$ and $P\left(x_{0}\right)$ have the same length and lie in the same side of $f(\mathbf{R})$ where $x_{0}=f_{ \pm 1}\left(t_{0}\right)$, and every half line centered at $x_{0}$ between $P\left(x_{0}\right)$ and $P^{\prime}\left(x_{0}\right)$ intersects $f(\mathbf{R})$. Such a function $P$ is easily constructed by starting from the function of shortest perpendiculars and changing it into intervals bounded by points of discontinuity which are not of types we required by choosing perpendiculars to the opposite side. For $x \in f_{ \pm 1}(\mathbf{R})$, let $H(x)$ be the length of $P(x)$. Then the following claim holds.

Claim 2. For any positive real number $\varepsilon$, there exists a constant $K$ such that $H\left(f_{ \pm 1}(t)\right)<\varepsilon$ if $t>K$.

Proof. We only consider $f_{1}$ because the proof for $f_{-1}$ is completely the same.

First we see that there does not exist a constant $\varepsilon$ such that for some $K, H\left(f_{1}(t)\right) \geq \varepsilon$ if $t \geq K$. Suppose that there were such $\varepsilon$. Then for each $i$ there would exist a constant $K_{i}$ such that the horizontal distance between $f_{i+1}(t)$ and $f_{i}(\mathbf{R})$ in one direction would be greater than $\varepsilon$ for $t>K_{i}$ by the assumption on $P(x)$. Then the horizontal distance between $f_{i}(t)$ and $f(\mathbf{R})$ would be greater than $i \varepsilon$ for $t>K_{i}$, which would contradict the assumption that the $x$ coordinate of $\bigcup_{i} f_{i}(\mathbf{R})$ is bounded.

Next we see that for any positive number $\varepsilon$, there exists a constant $K$ such that for any interval $[T, T+K]$ there exists $t \in[T, T+K]$ for which $H\left(f_{1}(t)\right)<\varepsilon$. Suppose that there were not such a constant $K$. Then there would exist $\varepsilon$, and for any $n \in \mathbf{N}$, there would exist intervals $\left[T_{n}, T_{n}+n\right]$ such that $H(f(t)) \geq \varepsilon$ if $t \in\left[T_{n}, T_{n}+n\right]$. Let $x()$ and $y()$ denote the $x$ and $y$-coordinate respectively. If $\left(\max _{t \in\left[T_{n}, T_{n}+n\right]} y(f(t))-\min _{t \in\left[T_{n}, T_{n}+n\right]} y(f(t))\right)$ does not go to $\infty$ as $n \rightarrow \infty$, some translates of $f\left[T_{n}, T_{n}+n\right]$ by some powers of $h$ converge to a non-proper map to $\lambda$ uniformly on a compact set because $x(f(t))$ is bounded, which is a contradiction. Hence we could obtain a sequence of intervals $\left\{\left[s_{n}, t_{n}\right]\right\}$ such that $y\left(f\left(t_{n}\right)\right)-y\left(f\left(s_{n}\right)\right)=n|h|$ and $H(f(t))>\varepsilon$ for $t \in\left[s_{n}, t_{n}\right]$ where $|h|$ denotes the translation length of $h$. Then the vertical distance between $f_{n}\left(s_{n}\right)$ and $f\left(t_{n}\right)$ would be greater than $n \varepsilon$, which is a contradiction.

Now if our claim does not hold, there must exist some $\varepsilon, K$ and intervals $I_{i}=\left[s_{i}^{0}, s_{i}^{1}\right]$ such that $\left|s_{i}^{1}-s_{i}^{0}\right| \leq K, H\left(f_{1}\left(s_{i}^{j}\right)\right)=\varepsilon / 2$, and 
$\max _{x \in I_{i}} H\left(f_{1}(X)\right) \geq \varepsilon$. It is obvious that $\left|s_{i}^{1}-s_{i}^{0}\right| \geq \varepsilon$. For $\varepsilon / 2$ let $J_{i}^{\varepsilon / 2}$ be an arc consisting of points on $P(f(x))$ at distance $\varepsilon / 2$ from $f(\mathbf{R})$ for $x \in I_{i}$ and circular arcs from $P\left(f\left(x_{0}\right)\right)$ to $P^{\prime}\left(f\left(x_{0}\right)\right)$ for all points $x_{0}$ in $I_{i}$ where $P \circ f$ is not continuous. (It is easy to see that such a set becomes an embedded arc.) Then by Claim 1 there exists a constant $\delta$ such that $\int_{J_{i}^{\varepsilon / 2}} d s \geq \delta$. For $\nu(\varepsilon / 2 \leq 3 \varepsilon / 4)$, let $I_{i}^{\nu}$ be the longest subarc of $I_{i}$ such that $H(f(x)) \geq \nu$ for every $x \in I_{i}^{\nu}$. The length of $I_{i}^{\nu}$ is at least $2(\varepsilon-\nu)$. We define $J_{i}^{\nu}$ for $I_{i}^{\nu}$ by the same way as we define $J_{i}^{\varepsilon / 2}$. Then the $J_{i}^{\nu}$ 's are disjoint for fixed $i$, and $\int_{J^{\nu}} d s \geq \delta$. Let $\delta^{\prime}$ be $\min \{\delta, \varepsilon / 4\}$, and let $\widetilde{J}_{i}^{\nu}$ be a subarc of $J_{i}^{\nu}$ with the length $\delta^{\prime}$ containing the initial endpoint of $J_{i}^{\nu}$. Let $S_{i}$ be their union $\bigcup_{\varepsilon / 2 \leq \nu \leq 3 \varepsilon / 4} \widetilde{J}_{i}^{\nu}$. Then Area $\left(S_{i}\right) \geq \varepsilon \delta^{\prime} / 4$. We can assume that the $S_{i}$ 's are disjoint by properness of $f_{1}$. If $S_{i}$ does not intersect $f_{j}(\mathbf{R})$ for $j \geq 2$ then $S_{i}$ is contained in $\bigcup_{j \leq k} D_{k}$. Therefore $S_{i}$ is disjoint from $h^{k}\left(S_{i^{\prime}}\right)$ for each $i^{\prime}$ and $k \geq j$. If $S_{i}$ intersects $f_{j}(\mathbf{R}), S_{j}$ is disjoint from $h^{j}\left(S_{i^{\prime}}\right)$ for every $i^{\prime}$ because $h^{j}\left(S_{i^{\prime}}\right)$ is apart from $f_{j}(\mathbf{R})$ with distance at least $\varepsilon / 2$ and $\operatorname{diam}\left(S_{i}\right)<\varepsilon / 2$. Thus as infinitely many $h^{j}\left(S_{i}\right)$ 's are contained in a compact set, it is a contradiction.

In the case of Fig. 3-B-(b), let $F_{i}^{+}: \mathbf{R}_{+} \rightarrow \lambda$ be a map whose image is the component of $D_{i-1} \cap f_{i}(\mathbf{R})$ containing the end, and let $F_{i}^{-}$be a map whose image is the component of $D_{i} \cap f_{i}(\mathbf{R})$ containing the end. Then by applying the same technique as Claim 2 we can prove that for each $\varepsilon$ there exists $K$ such that $d\left(F_{i}^{-}(t), F_{i}^{+}\left(\mathbf{R}_{+}\right)\right) \leq \varepsilon$ and $d\left(F_{i}^{+}(t), F_{i}^{-}\left(\mathbf{R}_{+}\right)\right) \leq \varepsilon$ for $t \geq K$.

As $f(\mathbf{R})$ is not an isolated leaf, there is a side of $f(\mathbf{R})$ such that for each perpendicular $p(t)$ at $f(t)$ to that side, $\int_{p(t)} \mu>0$. By Claim 2 and the remark above, there exists a side of $f(\mathbf{R})$ or $F^{+}\left(\mathbf{R}_{+}\right)$from which either $f_{1}(\mathbf{R})$ or $f_{-1}(\mathbf{R})$ or $F^{-}\left(\mathbf{R}_{+}\right)$approaches to $f(\mathbf{R})$ or $F^{+}\left(\mathbf{R}_{+}\right)$closer and closer. Assume that $f_{1}(\mathbf{R})$ approaches to $f(\mathbf{R})$. (Other cases are completely the same.) Let $\nu$ be the leaf of $\widetilde{L}$ which contains $f(\mathbf{R})$. Then for some $K$, the image $f_{1}([K, \infty))$ is contained in an $\eta$-neighbourhood $N_{\nu}$ of $\nu$. Suppose that $f_{1}([K, \infty))$ is contained in a leaf $\nu^{\prime}$ in $N_{\nu}$ different from $\nu$. Then along $f_{1}([K, \infty))$, $\nu^{\prime}$ approaches to $\nu$ closer and closer. Let $h_{t}$ be the interval between $f_{1}(t)$ and $\nu$ (on the side of $f(\mathbf{R})$ ) in the fiber containing $f_{1}(t)$ of the $I$-bundle structure of $N_{\nu}$. Then the leaves intersecting $h_{t_{0}}\left(t_{0}>K\right)$ remain to intersect $h_{t}$ for $t>t_{0}$ because they are transverse to all 
fibers of the $I$-bundle structure as is seen in the remark before we start the case (1). Therefore $\mu\left(h_{t}\right)$ (more precisely $\mu\left(q\left(h_{t}\right)\right)$ ) is constant as $t \rightarrow \infty$, and $\mu\left(h_{t}\right)>0$ because $\nu$ is not isolated on this side. As the length of $h_{t}$ with respect to $g$ goes to zero as $t \rightarrow \infty$, this contradicts Lemma 1.1 .

Next suppose that $f_{1}([K, \infty))$ is contained in $\nu$. We consider two cases in the following. Let $B_{t}$ be a flow box constituting $N_{\nu}$ which contains $f_{1}(t)$ and intersects $f(\mathbf{R})$ for sufficiently large $t$. The first case is when $f_{1}(t)$ is on a component of leaves different from that containing $f(\mathbf{R}) \cap B_{t}$ for every sufficiently large $t$, and the second case is when there is a sequence $\left\{t_{j}\right\}$ going to $\infty$ such that $f_{1}\left(t_{j}\right)$ is on the same component of leaves in $B_{t}$, as $f(\mathbf{R}) \cap B_{t_{j}}$. In the first case, we can consider the family of arcs $h_{t}$ as the paragraph before and it implies the same contradiction. In the second case, the distance between $f_{1}(t)$ and $f(\mathbf{R})$ on $\nu$ also goes to zero as $t \rightarrow \infty$. Consider the limit of the image of $\nu$ by $q$ along the region in $\nu$ bounded by $f_{1}(\mathbf{R})$ and $f(\mathbf{R})$ towards $+\infty$ direction. Then as the distance between $f_{1}\left(t_{j}\right)$ and $f(\mathbf{R})$ goes to zero on both $\lambda$ and $\nu$, the limit leaf must be tangent to $q(\lambda)$. It cannot be tangent to $q(\lambda)$ in the form of multiple saddle because the distance from $f_{1}(t)$ to $f(\mathbf{R})$ goes to zero. Hence the limit leaf is tangent to $q(\lambda)$ from one side. As $L^{\prime}$ is closed, this leaf is contained in $L^{\prime}$, hence is minimal surface. This is a contradiction.

At last we have proved that every embedding of type (1) must be $h$-invariant.

(2) Let $f: \mathbf{R} \rightarrow \lambda \cap \widetilde{L}^{\prime}$ be an embedding of type (2). Let $k$ be an element of $\pi_{1}(S)$ such that $\langle h, k\rangle=\pi_{1}(S)$, and such that $k$ translates $\lambda$ to positive direction with respect to the $x$-coordinate. Then for large $n$, both $f(\mathbf{R})$ and $h \circ f(\mathbf{R})$ intersect $h^{-n} \circ k \circ f(\mathbf{R})$ and $h^{-n-1} \circ k \circ f(\mathbf{R})$, which implies there exists a closed curve in $\lambda \cap \widetilde{L}^{\prime}$, a contradiction.

(3) Let $f$ be an embedding of type (3). Suppose that $f(\mathbf{R})$ and $h \circ f(\mathbf{R})$ intersect. Then there exists an $h$-invariant embedding $g$ from $\mathbf{R}$ into $\bigcup_{i=-\infty}^{\infty} h^{i} \circ f(\mathbf{R})$ of type (1). Then for some $k \in \pi_{1}(S)-\langle h\rangle$, $k \circ g(\mathbf{R})$ intersects each $h^{i} \circ f(\mathbf{R})$ transversely and they make a closed curve in $\lambda \cap \widetilde{L}^{\prime}$ together, which is a contradiction. Hence we can assume that $f(\mathbf{R}) \cap h \circ f(\mathbf{R})=\varnothing$. Moreover we can assume that for every $\gamma \in \pi_{1}(S), \gamma \circ f(\mathbf{R})$ does not intersect $f(\mathbf{R})$ transversely by changing the track of $f(\mathbf{R})$ at crossing point of $f(\mathbf{R}) \cap \gamma \circ f(\mathbf{R})$ is necessary. 
Let $E$ be one of the regions in $\lambda$ bounded by $f(\mathbf{R})$ which lies on the right side of $f(\mathbf{R})$ and only contains an end with $x \rightarrow \infty$. (We call this side the inside of $f(\mathbf{R})$.) Then for each $\gamma \in \pi_{1}(S)$, one of $\gamma(E) \cap E=\varnothing, \gamma(E) \subset E, \gamma(E) \supset E$ holds.

First assume that there exists a non-trivial element $k \in \pi_{1}(S)$ such that $k(E) \subset E$. Then there is a sequence of inclusions $\cdots \supset k^{-1}(E) \supset$ $E \supset k(E) \supset k^{2}(E) \supset \cdots$. As vertical diameter of $\bigcup_{i=-\infty}^{\infty} k^{i} \circ f(\mathbf{R})$ is bounded by $|h|$, the same argument as in the case of (1) can be applied, and we can see that for every $\varepsilon$ there exists $K$ such that $d\left(f(t), k^{ \pm 1} f(\mathbf{R})\right) \leq \varepsilon$ if $t \geq K$. As $f(\mathbf{R})$ is non-isolated from at least one side, the argument of (1) implies a contradiction.

Next assume that for each $\gamma \in \pi_{1}(S)-\{1\}, \gamma(E) \cap E=\varnothing$. We assume moreover that there exists a leaf of $\lambda \cap \widetilde{L}^{\prime}$ near $f(\mathbf{R})$ which is of type (3) and not isolated from the inside. As we have already excluded the cases (1) and (2), if such a leaf does not exist, almost all leaves near $f(\mathbf{R})$ are of type (4). This case will be dealt with in (4).

By retaking $f$, we can assume that $f(\mathbf{R})$ is not isolated from the inside. Let $W(t)$ be length $(E \cap\{x=t\})$. Then by the same argument as in (1), $W(t) \rightarrow 0$ as $t \rightarrow \infty$. But as $f(\mathbf{R})$ is not isolated from inside, the same argument as (1) implies that $\mu(E \cap\{x=t\})$ does not go to 0 as $t \rightarrow \infty$, which is a contradiction as in (1).

Thus such an embedding $f$ cannot exist.

(4) Suppose that there exists an embedding $f$ of type (4). We have already excluded the cases when there exists an embedding of type (1), (2), (3) except when there exists an embedding of type (3) such that all leaves near it are of type (4). Hence we can assume that there exists a neighbourhood of $f(\mathbf{R})$ containing a non-empty set of leaves of type (4). Let $n(x)$ be a short vertical line centered at $x$. Then for each $t \in \mathbf{R}, \mu(n(f(t)))>0$ because $f(\mathbf{R})$ is not isolated. On the other hand, $\mu[h]=0$. Let $\tilde{h}$ be a lift of a regular fiber on $\lambda$. Then for almost every leaf $l$ which intersects $\lambda$ near $f(\mathbf{R})$, the intersection of $\tilde{h}$ and $l$ is inessential. Hence each leaf $l$ intersects $\lambda$ near $f(\mathbf{R})$ by $m_{1}$ and again by $m_{2}$ where $l$ passes $\lambda$ to the opposite direction, and both $m_{1}$ and $m_{2}$ are of type (4). For almost every leaf $l$, we can assume that $l$ does not intersect the strip between $m_{1}$ and $m_{2}$ because almost all leaves of $\lambda \cap \widetilde{L}$ are of type (4). Then for almost every $l$, a union of parts of $\lambda$ and $l$ between $m_{1}$ and $m_{2}$ bounds a region homeomorphic to $D^{2} \times \mathbf{R}$. Hence we can choose such a region which is innermost. We use the notation $l, m_{1}, m_{2}$ for the ones whose bounding region is innermost. Then for the tie $T$ between $m_{1}$ 
and $m_{2}, \operatorname{int}(T) \cap \widetilde{L}=\varnothing$. Let $\tau$ be the tie on $l$ between $m_{1}$ and $m_{2}$. We will prove that the surfaces $q(T)$ and $q(\tau)$ are homeomorphic to the annulus. It is easy to see that if $q(T)$ is an annulus, $q(\tau)$ is also an annulus. Therefore we only need to show that $q(T)$ is an annulus. It is also easy to see that either $q \mid T$ is injective or $q(T)$ is an annulus. Let $v(t)$ be the vertical width of $T$ at $x=t$. If $v(t)$ does not go to 0 as $t \rightarrow \pm \infty$, the surface $q(T)$ must be an annulus because $q(T)$ is a subsurface of the surface $S$ which has a finite area. If $v(t)$ goes to 0 as $t \rightarrow \pm \infty$, let $v^{\prime}(t)$ be the distance on $\tau$ of the two intersections of $\partial T$ and $x=t$. Suppose $v^{\prime}(t)$ does not go to 0 as $t \rightarrow \pm \infty$. Then the closure of $q(\tau)$ must contain a point where $L^{\prime}$ is tangent to itself from one side, because we can choose a sequence of the two intersections of $x=t$ and $\partial T=\partial \tau$ whose image by $q$ converges to the same point. If $v^{\prime}(t)$ goes to 0 as $t \rightarrow \infty, q(\tau)$ converges to a surface which is tangent to $S$ from one side. Hence $q(T)$ must be an annulus.

Then there exists a point $P$ in int $\tau$ where the distance function from $\tau$ to $\lambda$ attains the maximum. By translating $\tau$ along the perpendicular from $P$ to $\lambda$ by an isometry $\zeta$, the surface $\zeta(\tau)$ becomes tangent to $\lambda$ from one side, which is a contradiction. This completes the proof of the case (4).

Now let us consider the intersection $\widetilde{L} \cap \widetilde{L}^{\prime}$. As $L$ is a vertical set over a measured geodesic lamination on $O, L$ is approximated by vertical tori. More precisely, for each point $x \in L$ and $\varepsilon>0$, there exists a vertical torus on either side of $L$ which passes through an $\varepsilon$-neighbourhood of $x$ and whose tangent plane at that point differs at most $\varepsilon$ from the tangent plane of $L$ at $x$.

Let $\lambda$ be a component of $\widetilde{L}$. Now suppose that there exists a component of $\lambda \cap \widetilde{L}^{\prime}$ which is not of type (1) and $h$-invariant. We can prove that there are no components of type (1) which are not $h$ invariant by the same argument as for $\widetilde{S} \cap \widetilde{L}^{\prime}$. As for each nearby torus $S, \widetilde{S} \cap \widetilde{L}^{\prime}$ is of type (1) and $h$-invariant, and for each point $x \in L, L$ passes arbitrarily near $x$ infinitely many times, there exist infinitely many components of $\lambda \cap \widetilde{L}^{\prime}$ of type (1), which make closed curves in $\lambda \cap \widetilde{L}^{\prime}$ with a component of type (2), (3), (4), a contradiction. Hence $\lambda \cap \widetilde{L}^{\prime}$ also consists of components which are of type (1) and $h$-invariant.

Next we will prove that actually each component of $L^{\prime}$ is either contained in $L$ or disjoint from $L$. Assume that there exists an embedding $f: \mathbf{R} \rightarrow \lambda \cap \widetilde{L}^{\prime}$ which is $h$-invariant. Let $\nu$ be the leaf 
of $\widetilde{L}^{\prime}$ containing $f(\mathbf{R})$. Let $z$ be a point on $f(\mathbf{R})$ such that there exists a horizontal interval $I$ centered at $z$ which is transverse to $\lambda \cap \widetilde{L}^{\prime}$. As we have assumed that no leaves are isolated, $\mu^{\prime}(I)=\varepsilon>0$. Recall that $L$ is a saturated set over a measured geodesic lamination $l$. Let $l_{0}$ be a component of $l$. Then each leaf of $l_{0}$ is dense in $l_{0}$. Hence there exists a sequence of simple closed curves $\left\{\sigma_{i}\right\}$ containing $p \circ q(z) \in O$, consisting of an arc on $l_{0}$ one of whose ends is $p \circ q(z)$ and an arc transverse to $l_{0}$ with length $1 / i$. Then $i\left(l_{0}, \sigma_{i}\right) \rightarrow 0$, where $i$ denotes the geometric intersection number on $O$. Let $s_{i}$ be a lift of $\sigma_{i}$ to $M$. Then $\mu\left(s_{i}\right) \rightarrow 0$. Let $\tilde{s}_{i}$ be a lift of $s_{i}$ to $\widetilde{M}$. We can take $s_{i}$ and $\tilde{s}_{i}$ so that $\tilde{s}_{i}$ contains the half of $I$ which has positive $\mu^{\prime}$-measure. Recall that every component of $\lambda \cap \widetilde{L}^{\prime}$ is of type (1) and $h$-invariant. Then as $\left[L^{\prime}, \mu^{\prime}\right]\left(\left[s_{i}\right]\right) \leq[L, \mu]\left(\left[s_{i}\right]\right)$ by the assumption and the right hand goes to zero as we saw above, there is an intersection of $\tilde{s}_{i}$ and $\widetilde{L}^{\prime}$ near $z$ which is inessential. Let $f^{\prime}: \mathbf{R} \rightarrow \lambda \cap \widetilde{L}^{\prime}$ be the inessential intersection and let $\nu^{\prime}$ be the leaf of $\widetilde{L}^{\prime}$ containing $f^{\prime}(\mathbf{R})$. Then $\lambda \cap \nu^{\prime}$ contains another component $f^{\prime \prime}(\mathbf{R})$ such that $f^{\prime}(\mathbf{R})$ and $f^{\prime \prime}(\mathbf{R})$ bounds $R \cong I \times \mathbf{R}$ on $\nu^{\prime}$. Then $R /\langle h\rangle$ is a compact annulus, and $\lambda /\langle h\rangle$ is an open annulus which is a closed set in $\widetilde{M} /\langle h\rangle$. The distance function from $R /\langle h\rangle$ to $\lambda /\langle h\rangle$ lifts to that from $R$ to $\lambda$. Hence the distance function $d$ to $\lambda$ is bounded on $R$. Therefore $d$ attains the maximum at some point $r$ on $R$. We translate $\nu$ by an isometry $\zeta$ along the perpendicular from $r$ to $\lambda$ so that $\zeta(\nu)$ is tangent to $\lambda$ from one side, which is a contradiction. Now we have proved that $L$ does not intersect $L^{\prime}$ transversely, which implies that each component of $L^{\prime}$ is either contained in $L$ or disjoint from $L$.

Next we will prove that each leaf of $L^{\prime} \backslash L$ is vertical. Suppose that $L^{\prime} \backslash L \neq \varnothing$. Then as each component of $L^{\prime}$ is either contained in $L$ or disjoint from $L$, there exists a leaf $l$ of $L^{\prime}$ contained in $M-L$. Each component of $M-L$ is a Seifert fibered manifold whose base space is an open hyperbolic 2-orbifold and can be completed to a Seifert fibered manifold $N$ whose base space is a complete hyperbolic 2-orbifold with totally geodesic boundaries. As $L^{\prime}$ is carried by an incompressible branched surface, if $l$ is not an image of a properly embedded open annulus or a torus which is parallel to a boundary component, there is a loop $\sigma$ in $M-L$ which intersects $l$ essentially. In that case, $i\left(\sigma, L^{\prime}\right) \neq 0$ and $i(L, \sigma)=0$ which contradicts the assumption that $[L]=\left[L^{\prime}\right]$ in $\mathbf{R}^{\mathscr{S}}$. If $l$ is a torus, it must coincide with a boundary component because $l$ is minimal surface, which contradicts the assumption that $l \subset M-L$. Assume that $l$ is 
a properly embedded open annulus. Then its ends extend to cusps. Let $\widetilde{N}$ be a lift of $N$ to $\widetilde{S L_{2}} \mathbf{R}$ and let $\tilde{l}$ be a lift of $l$ to $\widetilde{N}$. Then $\widetilde{N}$ is a saturated set over an ideal polygon $P$ with possibly infinitely many sides. We call a saturated set over cusps of $P$ also cusps. Then $\tilde{l}$ is a plane joining two different cusps of $\widetilde{N}$. Let $a, b$ be two cusps of $P$ corresponding to these cusps. Let $\Pi$ be the saturated plane in $\widetilde{N}$ over the geodesic in $P$ joining $a$ and $b$, and suppose that $\tilde{l} \neq \Pi$. Let $d$ be the distance function on $\tilde{l}$ to $\Pi$. Then the value $d(x)$ goes to 0 as $x$ goes to cusps, and $d$ is a lift of a distance function on $l$. Hence the function $d$ is bounded and there is a point $x_{0} \in \tilde{l}$ where $d$ attains the maximum. Then by the same argument as before, we can translate $\tilde{l}$ by an isometry so that it is tangent to $\Pi$ from one side, which is a contradiction. Now we have proved that each leaf of $L^{\prime} \backslash L$ is vertical.

Thus we have proved that $L^{\prime}$ is also vertical with respect to the geometric structure $g$ on $M$. Hence $L^{*}$ is also a saturated set over a geodesic measured lamination on $O$. As the geodesic measured laminations on a hyperbolic 2-orbifold $O$ which have the same image in $\mathbf{R}^{\mathscr{E}}$ (where $\mathscr{C}$ denotes the set of a free homotopy class of $\pi_{1}^{\text {orb }}(O)$ ) must coincide, the projections of $L$ and $L^{\prime}$ to the base orbifold $O$ coincide by the assumption that $[L]=\left[L^{*}\right]$ in $\mathbf{R}^{\mathscr{S}}$. Therefore $L$ and $L^{*}$ must coincide.

By the same argument we can prove the following

THEOREM 3.2. Let $M$ be a Seifert fibered manifold with a fixed $H^{2} \times E$-structure $g$. Let $m$ be a class of $\mathbf{R}^{\mathscr{S}}$ represented by an incompressible measured lamination such that $m(h)=0$ where $h$ denotes the class of regular fibers. Then there exists a unique incompressible minimal measured lamination $L$ in $(M, g)$ which represents $m$. Moreover $L$ is vertical with respect to geometric fibration of $(M, g)$.

The following corollary is obtained by the theorems above and Corollary 2.3.

Corollary 3.3. Let $(M, g)$ be as in Theorem 3.1. Then every incompressible measured lamination is equivalent to a unique minimal measured lamination. Moreover every minimal measured lamination is a vertical saturated set over some geodesic measured lamination on the base 2-orbifold $O$. 


\section{REFERENCES}

[1] A. Casson and A. Bleiler, Automorphisms of surfaces after Nielsen and Thurston, London Math. Soc. Student Texts, 9, Cambridge Univ. Press.

[2] M. Freedman, P. Scott and J. Hass, Least area incompressible surfaces in 3manifolds, Inv. Math., 71 (1983), 609-642.

[3] W. Floyd and U. Oertel, Incompressible surfaces via branched surfaces, Topology, 23 (1984), 117-125.

[4] J. Hass, Minimal surfaces in Seifert fiber spaces, Topology Appl., 18 (1984), 145-151.

[5] J. Morgan and P. Shalen, Degeneration of hyperbolic structures II: Measured laminations in 3-manifolds, Ann. Math., 127 (1988), 403-456.

Received October 18, 1988 and in revised form May 8, 1989.

TOKyo Metropolitan University

Fukasawa, Setagaya, Tokyo 158

JAPAN

Current address: Tokyo Institute of Technology

Ôokayama, Meguro, Tokyo 152

Japan 


\section{PACIFIC JOURNAL OF MATHEMATICS EDITORS}

\author{
V. S. VARADARAJAN \\ (Managing Editor) \\ University of California \\ Los Angeles, CA 90024-1555-05 \\ Herbert Clemens \\ University of Utah \\ Salt Lake City, UT 84112 \\ THOMAS ENRIGHT \\ University of California, San Diego \\ La Jolla, CA 92093
}

R. FINN

Stanford University

Stanford, CA 94305

Hermann Flaschka

University of Arizona

Tucson, AZ 85721

VAUGHAN F. R. Jones

University of California

Berkeley, CA 94720

Steven Kerckhoff

Stanford University

Stanford, CA 94305

\author{
C. C. MOORE \\ University of California \\ Berkeley, CA 94720
}

MARTIN SCHARLEMANN

University of California

Santa Barbara, CA 93106

HAROLd STARK

University of California, San Diego

La Jolla, CA 92093

\section{ASSOCIATE EDITORS}
R. ARENS
E. F. BECKENBACH
B. H. NeUmanN
F. WoLF
(1904-1989)
K. YosHIDA
(1906-1982)

\section{SUPPORTING INSTITUTIONS}

UNIVERSITY OF ARIZONA
UNIVERSITY OF BRITISH COLUMBIA
CALIFORNIA INSTITUTE OF TECHNOLOGY
UNIVERSITY OF CALIFORNIA
MONTANA STATE UNIVERSITY
UNIVERSITY OF NEVADA, RENO
NEW MEXICO STATE UNIVERSITY
OREGON STATE UNIVERSITY

UNIVERSITY OF ARIZONA

CAIVERSITY OF BRITISH COLUN UNIVERSITY OF CALIFORNIA

MONTANA STATE UNIVERSITY OREGON STATE UNIVERSITY

\author{
UNIVERSITY OF OREGON \\ UNIVERSITY OF SOUTHERN CALIFORNIA \\ STANFORD UNIVERSITY \\ UNIVERSITY OF HAWAII \\ UNIVERSITY OF TOKYO \\ UNIVERSITY OF UTAH \\ WASHINGTON STATE UNIVERSITY \\ UNIVERSITY OF WASHINGTON
}

The Supporting Institutions listed above contribute to the cost of publication of this Journal, but they are not owners or publishers and have no responsibility for its content or policies.

Mathematical papers intended for publication in the Pacific Journal of Mathematics should be in typed form or offset-reproduced (not dittoed), double spaced with large margins. Please do not use built up fractions in the text of the manuscript. However, you may use them in the displayed equations. Underline Greek letters in red, German in green, and script in blue. The first paragraph must be capable of being used separately as a synopsis of the entire paper. In particular it should contain no bibliographic references. Please propose a heading for the odd numbered pages of less than 35 characters. Manuscripts, in triplicate, may be sent to any one of the editors. Please classify according to the 1980 Mathematics Subject Classification (1985 Revision) scheme which can be found in the December index volumes of Mathematical Reviews. Supply name and address of author to whom proofs should be sent. All other communications should be addressed to the managing editor, or Elaine Barth, University of California, Los Angeles, California 90024-1555-05.

There are page-charges associated with articles appearing in the Pacific Journal of Mathematics. These charges are expected to be paid by the author's University, Government Agency or Company. If the author or authors do not have access to such Institutional support these charges are waived. Single authors will receive 50 free reprints; joint authors will receive a total of 100 free reprints. Additional copies may be obtained at cost in multiples of 50 .

The Pacific Journal of Mathematics (ISSN 0030-8730) is published monthly. Regular subscription rate: $\$ 190.00$ a year (12 issues). Special rate: $\$ 95.00$ a year to individual members of supporting institutions.

Subscriptions, orders for numbers issued in the last three calendar years, and changes of address should be sent to Pacific Journal of Mathematics, P.O. Box 969, Carmel Valley, CA 93924, U.S.A. Old back numbers obtainable from Kraus Periodicals Co., Route 100, Millwood, NY 10546.

The Pacific Journal of Mathematics at P.O. Box 969, Carmel Valley, CA 93924 (ISSN 0030-8730) is published monthly. Second-class postage paid at Carmel Valley, California 93924, and additional mailing offices. Postmaster: send address changes to Pacific Journal of Mathematics, P.O. Box 969, Carmel Valley, CA 93924.

\section{PUBLISHED BY PACIFIC JOURNAL OF MATHEMATICS, A NON-PROFIT CORPORATION}




\section{Pacific Journal of Mathematics}

Vol. 144, No. $2 \quad$ June, 1990

George E. Andrews and David M. Jackson, An algebraically derived $q$-analogue of a character sum associated with a class of semiregular

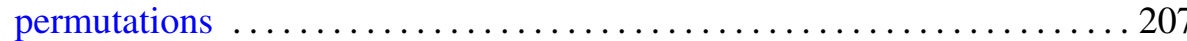

Fabio Bardelli and Andrea Del Centina, The moduli space of genus four double covers of elliptic curves is rational $\ldots \ldots \ldots \ldots \ldots \ldots \ldots \ldots 219$

Young Do Chai, An estimate of the volume of a compact set in terms of its

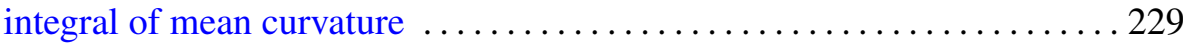

Salvador Comalada, Elliptic curves with trivial conductor over quadratic fields

Kahar El-Hussein, Résolubilité semi-globale des opérateurs différentiels invariants sur les groupes de déplacements $\ldots \ldots \ldots \ldots \ldots \ldots \ldots \ldots . \ldots 259$

David M. Goldschmidt, Classical link invariants and the Burau representation

Liliana Janicka, Radon-Nikodým problem for the variation of a vector measure

Wacław Marzantowicz, An almost classification of compact Lie groups with Borsuk-Ulam properties ............................ 299

Akira Ohbuchi, On the projective normality of some varieties of degree 5 . . 313 Ken'ichi Ohshika, Minimal measured laminations in geometric

3-manifolds

Hal Leslie Smith, A discrete Lyapunov function for a class of linear differential equations

John Samuel Spielberg, Diagonal states on $O_{2} \ldots \ldots \ldots \ldots \ldots \ldots \ldots \ldots$

Thomas Vogel, A note on the sessile drop

Gerold Wagner, On means of distances on the surface of a sphere (lower bounds) 